\title{
No. VIII.-ISOPTERA.
}

Von Nils F. Holmgren, Рн.D., Dozent an der Hochschule in Stockholm.

(Mittgeteilt von Prof. J. Stanley Gardiner, M.A., F.R.S., F.L.S.)

Gelesen den 17. Februar 1906.

Die Kenntnis des madagassischen Faunengebietes hat immer und mit Recht grosses Interesse beansprucht und man kann wohl sagen dass es faunistisch recht wohl bekannt geworden ist. Auch die Termitenfauna dieses Gebietes ist einigermassen studiert worden, ist aber jedoch verhältnismässig wenig erforscht geblieben. Die ersten ausführlichen Mitteilungen über die Termiten Madagaskars verdanken wir dem unermüdlichen Ameisen- und Termitenforscher Pater E. Wasmann, der von diesem Gebiet zehn Arten beschrieben hat. Später hat Sjöstedt die Faunakunde mit sieben neuen Arten vermehrt. Dazu kommen dann noch zwei von mir beschriebene Arten und der wohl nicht mehr identificierbare Calotermes pallidus (Ramb.). Ausserdem können hierzu Termes bellicosus Smeath. und Termes natalensis Hav. gerechnet werden, welche jedoch wohl von Afrika eingeschleppt sind. Die Mehrzahl der echt madagassischen Arten ist auf der madagassischen Haupt-Insel gefunden. An den kleineren Inselgruppen in der madagassischen Region sind nur folgende Arten angetroffen. Auf Aldabra: Eutermes salebri thorax Sjöst. und Microcerotermes subtilis Wasm. Auf Mauritius: Calotermes hova Wasm., Calotermes pallidus (Ramb.) und Eutermes mauricianus Sjöst. Von diesen letzterwähnten Arten kommen folgende ausserdem auf Madagaskar vor: Calotermes hova, Microcerotermes subtilis und Eutermes salebrithorax.

Aus dieser Übersicht geht also zweifellos hervor wie erwünscht eine Erweiterung unsrer Kenntnis des fraglichen Faunengebietes ist. Besonders die kleineren madagassischen Inselgruppen waren bis jetzt in termitologischer Hinsicht wenig untersucht worden. Die von Mr Hugh Scott auf den Seychellen und von Mr J. C. F. Fryer auf Aldabra zusammengebrachte Sammlung muss deshalb von grossem Interesse sein, besonders weil sie von den nördlichsten der Inselgruppen, den Seychellen und den Admiranten grösstenteils stammt, von denen noch keine Termiten bekannt waren.

Die Sammlung enthält 9 Arten und Unterarten, von denen 5 neu sind. Von den früher bekannten, wird die Kenntnis von 3 durch Entdeckung von den früher unbekannten Geschlechtsindividuen komplettiert.

1. Calotermes laticollis, n. sp. Imagines, Soldaten.

2. " (Procryptotermes) fryeri, n. sp. Imagines, Soldaten, Larven.

3. , (Glyptotermes) scotti, n. sp. Imagines, Soldaten, Larven.

4. $\quad$ (s. lat.) longus, n. sp. 2 Soldaten.

5. Arrhinotermes canalifrons (Sjöst.) Neotenen, Soldaten, Arbeiter. 
6. Coptotermes truncatus Wasm. Nymphen, Soldaten, Arbeiter.

7. Microcerotermes subtilis Wasm. Imagines, Soldaten, Arbeiter.

8. Eutermes nigrita Wasm. Imagines, Nymphen, Soldaten, Arbeiter.

9. Eutermes nigrita subsp. mahéensis, n. subsp. Nymphen, Soldaten, Arbeiter.

Hinsichtlich des Verhältnisses des madagassischen Faunengebietes zu dem kontinentalafrikanischen scheinen die Termiten kein bestimmtes auszusagen. Die meisten madagassischen Gattungen sind auch in Afrika vorhanden, aber gemeinsame Arten fehlen in grosser Ausdehnung, und wenn solche Arten vorhanden sind, so sind sie wahrscheinlich eingeschleppt. Interessant ist jedoch dass die Calotermes-Untergattungen Procryptotermes und Glyptotermes noch nicht in Afrika angetroffen sind, ebenso dass die Gattung Arrhinotermes da noch nicht endeckt ist.

Das Subgenus Procryptotermes kommt aber in Ost-Indien mit der Art P. domesticus (Hav.) vor. Das damit nahe verwandte Subgenus Cryptotermes gehört Amerika zu, wo Banks ein Cryptotermes cavifrons von Florida beschrieben hat. Ausserdem befinden sich in meiner Sammlung und im Berliner Museum zwei noch unbeschriebene Arten aus Brasilien, gesammelt von W. Müller. Vielleicht ist auch Calotermes brevis Hagen aus Brasilien ein Procryptotermes. Calotermes braueri Frogg. aus Australien gehört auch vielleicht diesem Verwandtschaftskreis an.

Das Subgenus Glyptotermes kommt ausser in dem madagassischen Gebiet auch in Indien mit einigen Arten, z. B. G. borneensis Hav, vor. Aus Australien sind vier Arten von Froggatt beschrieben worden, und in Süd-Amerika habe ich eine noch unbeschriebene Art entdeckt.

Die Verbreitung dieser beiden Subgenera scheint somit ungefähr dieselbe zu sein.

Arrhinotermes, die von Wasmann aufgestellt wurde, kommt ausserhalb Madagascar auch in Vorderindien mit $A$. heimi Wasm., auf die Jaluit-Atolle (eine noch unbeschriebene Art), auf den Cocos-Inseln zwischen den Galapagos Inseln und Costa Rica mit $A$. oceanicus Wasm., und in Costa Rica (eine noch unbeschriebene Art) vor*.

Arrhinotermes besitzt also wahrscheinlich eine ähnliche Verbreitung wie die beiden obigen Subgenera.

Bemerkenswert ist es, dass die madagassischen Gattungen, welche in kontinentalen Afrika vertreten sind, auch in Indien vorkommen wie: Calotermes, Capritermes, Microcerotermes, Mirotermes, Coptotermes und Eutermes (und Microtermes?).

Hingegen scheinen die in sowohl Afrika wie Indien vertretenen Gattungen Hodotermes, Rhinotermes, Leucotermes, Synacanthotermes, Acanthotermes, Termes, Hamitermes, Eurytermes, auf Madagascar zu fehlen.

Noch ist es zu früh irgend welche positive Schlussfolgerungen aus den oben erwähnten Verhältnissen zu ziehen. Die Tatsachen scheinen aber darauf hinzudeuten, dass die madagassische Termitenwelt mehr mit dem indischen übereinstimmt als mit dem afrikanischen. Dass Afrika und Madagascar gemeinsame Gattungen besitzen lässt sich so erklären, dass eine Einwanderung vom Norden (Indien) aus sowohl nach Afrika wie nach Madagascar einst stattgefunden hat, und dass diese nicht ganz gleichzeitig gewesen. Aber

* Hierzu kommt eine als Prorhinotermes, n.g., bezeichnete Art, welche Silvestri neuerdings aus Samoa beschrieben hat. 
auf dem heutigen Standpunkt unsrer Kenntnis bleiben diese Erwägungen nur vage Hypotesen.

Jedoch trägt Scott's Sammlung ohne Zweifel dazu bei, die tiergeographischen Verhältnisse des madagassischen Faunengebietes zu klären.

Die hier unten benutzte systematische Aufstellung ist aus einem noch nicht publicierten, neuen Termitensystem, dass ich später veröffentlichen werde, herausgegriffen.

Bei Identificieren der Arten habe ich ausser meiner eigenen Sammlung auch die Sammlungen des hiesigen Reichsmuseums benutzt. Für das diesbezügliche Entgegenkommen des Museum-Präfekts, Herr Professor Y. Sjöstedt, spreche ich hier meinen besten Dank aus. Ebenso ist es mir eine angenehme Pflicht, dem Herrn Professor J. Stanley Gardiner (Cambridge), deshalb herzlich zu danken, weil er mir in der Lage versetzt hat, diese interessante Seychellen-sammlung zu bearbeiten. Ebenso danke ich Herrn Hugh Scott hier auch für Mitteilung von zahlreichen indischen Termiten-Arten, welche als werthvolles Vergleichmaterial bei den vorliegenden Studien gedient haben.

Fam. Protermitidæ, nov. fam.

Vorläufige Diagnose: Fontanell mit Fontanelldrüse fehlt immer. Praefrons kurz, dreieckig, Antennale bandförmig sich quer über dem Kopf streckend. Malpighische Gefässe 8. Hinterdarmsabteilung I.-II. kurz.

Diese neue Familie umfasst folgende Subfamilien: Mastotermitinæ Desn., Hodotermitinæ, n. subfam., Stolotermitinæ, n. subfam., und Calotermitinæ, n. subfam.

\section{Subfam. Calotermitinæ, n. subfam. \\ Gattung Calotermes Hagen.}

Untergattung: Calotermes (s. str.) Holmgr., nov. subgen.

1. Calotermes laticollis, n. sp.

Imago: Gelb-rotbraun. Hinterleib hell geringt, Unterseite weissgelb. Letzte Sternite braun. Flügel graubraun mit dunkleren Randrippen.

Kopf, Pronotum und Beine sehr dünn behaart. Abdominaltergite und Sternite mit Behaarung besonders auf ihrer hinteren Hälfte.

Kopf ziemlich dick, breit eiförmig, hinter den Augen beinahe halbcirkelförmig abgerundet, vor den Augen ein wenig verschmälert. Facettaugen mittelgross, ein wenig dorsal gelagert. Ozellen ziemlich gross, unmittelbar an den Augen gelegen. Kopfnähte deutlich, fein. Vorderrand des Transversalbandes schwach konkav. Basalteil des Clypeus kurz, Apicalteil länger. Oberlippe stark geneigt, schalenförmig. Antennen 20-21-gliedrig. 2. Glied so lang wie 3.; 4. bedeutend kürzer. 5. ein wenig länger als 4 .

Pronotum bedeutend breiter als der Kopf zwischen den Augenspitzen, nicht so lang wie die halbe Breite. Vorderrand breit konkav; Hinterrand konvex, in der Mitte schwach ausgerandet. Meso- und Metanotum mit beinahe geradem Hinterrand. 
Die Subcosta der Vorderflügel streckt sich über den inneren Drittel des Flügels und besitzt einen kurzen, basalen Ast. Radius einfach, streckt sich ein wenig über die Mitte des Flügels. Radius sector erstreckt sich zu der Flügelspitze, parallel mit und dem Radius ziemlich genähert. Er besitzt 6 Zweige zu dem Vorderrand des Flügels. Von diesen sind die 4 inneren lang (der 1. der längste und die übrigen von abnehmender Länge). Die Mediana läuft, stark markiert, parallel mit dem rad. sector bis zu der Spitze des Flügels und ist mit diesen vermittelst 12-14 kurzen Querrippen vereint. Die Flügelmembran ist zwischen der Mediana und dem Cubitus reticuliert. Cubitus mit ungef. 12 Ästen zu dem Hinterrande des Flügels, von denen jedoch nur die 6 à 7 inneren deutlich markiert sind.

Die Mediana der Hinterflügel entspringt vom rad. sector, ein wenig von seiner Basis. Die Distanz zwischen den parallelverlaufenden Rippen ist länger als gewöhnlich bei Calotermes. Tarsen je mit einem wohl entwickelten Onychium.

Länge mit Flügeln $19 \mathrm{~mm}$.

" ohne Flügeln 12-13 mm.

, des Kopfes mit Oberlippe $2 \cdot 4 \mathrm{~mm}$.

„ des Kopfes zu dem Vorderrande des Transversalbandes $1.7 \mathrm{~mm}$.

Breite des Kopfes $2 \cdot 1 \mathrm{~mm}$.

Soldat. Kopf gelbbraun, vorn ein wenig dunkler. Mandibelbasis braun; Mandibeln im übrigen schwarz. Körper strohgelb.

Kopf, Thorax (und Beine) dünn behaart. Abdominaltergite und Sternite mit zwei transversalen Borstenreihen, eine mittlere und eine hintere.

Kopf gross, Kopfseiten schwach abgerundet. Augen klein, rudimentär. Antennleisten mit einer schwachen ohrförmigen Verbreitung. Mandibelkondylen gross. Clypeus zieml. schmal, kurz; Spitzteil unbedeutend. Oberlippe kurz. Antennen 16-gliedrig; 3. Glied ein wenig länger als 2., dunkler als die übrigen Glieder. 4. Glied unbedeutend kürzer als 2. Mandibeln kräftig, gleichförmig gekrümmt. Die 'linke mit 5 kleineren Zähnen innerhalb der Spitze; die rechte mit einem grossen dreieckigen Zahn an der Mitte und mit einem kräftigen Basalzahn.

Pronotum (bedeutend) breiter als der Kopf, viel kürzer als die halbe Breite. Vorderrand winkelig konkav; Hinterrand beinahe gerade. Mesonotum und Metanotum bedeutend schmäler als Pronotum. Cerci kurz; Styli wohl entwickelt. Klauen und Tibialdornen braun.

Körperlänge $12-13 \mathrm{~mm}$.

Länge des Kopfes mit Mandibeln $6 \mathrm{~mm}$.

Breite des Kopfes $3 \mathrm{~mm}$.

Länge der Mandibeln $2 \mathrm{~mm}$.

Nymphe: Länge des Körpers $11 \mathrm{~mm}$.

, der Flügelscheiden $4 \mathrm{~mm}$.

Larve mit kleinsten Flügelscheiden (arbeiterähnlich) :

Länge des Körpes $8 \mathrm{~mm}$.

Flügelscheiden kaum mehr als angedeutet. 
Larve mit mittelgrossen Flügelscheiden:

Länge des Körpers $10.5 \mathrm{~mm}$.

, der Flügelscheiden $1.5 \mathrm{~mm}$.

Larve mit grossen Flïgelscheiden (Nymphenähnlich):

Länge des Körpers $8 \mathrm{~mm}$.

„, der Flügelscheiden $3 \mathrm{~mm}$.

"Arbeiter."

Länge des Körpers $11 \mathrm{~mm}$.

Ohne Flügelscheiden.

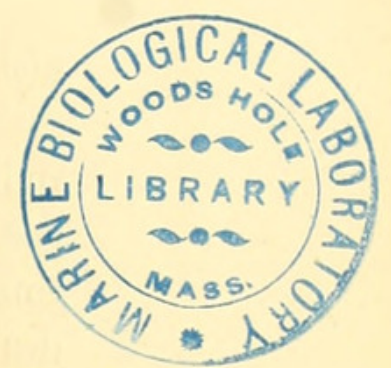

Bemerkungen: Diese Art ist durch ihren breiten Prothorax und vielgliedrigen Antennen sehr charakteristisch. Keine der bereits bekannten Calotermesarten besitzen so viel wie 21-gliedrige Antennen.

Fundorte. Seychellen. Mahé; nahe Morne Blanc, ungef. 800 Fuss Höhe, von einem Nest in einem gefallenen Baumstamm, X. 1908 (alle Stände); Long Island, VII. 1908 (alle Stände). Silhouette: Wald oberhalb Mare aux Cochons, mehr als 1000 Fuss o. M., aus einem gefallenen Ast, IX. 1908 (Soldaten und Larven).

Untergattung Procryptotermes Holmgr., nov. subgen.

2. Calotermes (Procryptotermes) fryeri, n. sp.

Imago: Hell graubraun.

Sehr kurze Haare an der Stirn, am Vorder- und Hinterrande des Pronotums, an den Hinterrändern der Abdominalplatten. Abdominalsterniten hinten mit einer Reihe von längeren Borsten.

Kopf ziemlich breit, oval, hinter den Augen halbcirkelförmig abgerundet. Augen ziemlich gross, wenig ausstehend. Ozellen mittelgross, unmittelbar an den Augen gelegen. Vorderrand des Transversalbandes ziemlich gerade. Clypeus mit wenig entwickeltem Basalteil; Spitzteil deutlich, hyalin. Oberlippe stark geneigt und gewölbt. Antennen 16-gliedrig. 2. und 3. Glied ungef. gleich lang. 4. Glied kürzer und so lang wie $5 . ; 6$. so lang wie 3.

Pronotum so breit wie der Kopf zwischen den Augenspitzen. Vorderrand schwach konkav ; Seitenränder schwach bogenförmig; Hinterecken stark abgerundet; Hinterrand in der Mitte schwach eingebuchtet. Meso- und Metanotum hinten beinahe gerade.

Flügel dünn, hyalin. Costa und subcosta, radius und radius sector stark markiert, bräunlich. Radius sector ist mit dem Radius mittelst 4 ziemlich langen, schiefgestellten Ästen verbunden. Die Mediana vereint sich, bogenförmig, mit dem radius sector in dessen äusserer Hälfte. Der Cubitus ist äusserst schwach markiert, kaum sichtbar. Tibien mit drei Spitzdornen.

Körperlänge mit Flügeln $9-10 \mathrm{~mm}$.

, ohne Flügel $5-6 \mathrm{~mm}$.

Länge des Kopfes, mit Oberlippe $1 \cdot 16 \mathrm{~mm}$.

" " $" \quad$ vom Vorderrande des Transversalbandes $1.05 \mathrm{~mm}$.

Breite des Kopfes $1.05 \mathrm{~mm}$. 
Soldat. Kopf gelbbraun, vorn dunkler; Oberkiefer pechschwarz. Körper strohgelb mit rostgelblichem Farbenton.

Behaarung in der Hauptsache wie bei der Imago. Kopf- und Hinterleibseiten jedoch dichter und länger behaart.

Kopf rectangulär mit abgerundeten Hinterecken und geradem Hinterrand. Augen rudimentär. Von den Augen aus verschmälert sich der Kopf ein wenig. Von der Seite gesehen ist der Kopf wie schief abgeschnitten. Vorderteil der Scheitel ist deutlich rinnenförmig vertieft. Die Rinne erweitert sich nach vorn. Stirnteil (Transversalband) des Kopfes flasch vertieft.

Vorderecken der Antennleisten sind ausserhalb der medialen Mandibelkondylen processenartig stark hervorgezogen. Die Kopfseiten ausserhalb der lateralen Mandibelkondylen sind auch processenartig. Clypeus bandförmig mit schwachem Spitzteil. Oberlippe ganz kurz. Mandibeln basal auf der äusseren Seite deutlich aufgetrieben, lang, ziemlich gerade, mit eingebogenen Spitzen. Nur basal giebt es schwach angedeutete Zähne. Antennen sehr kurz, 10-gliedrig. 3. Glied mehr als doppelt so lang wie 2., basal schmal, apical stark aufgetrieben, mehr als doppelt so dick wie basal. 4. - 10 . Glieder ganz kurz, schmäler als die vorhergehenden. 10. Glied eiförmig, schmäler als die vorhergehenden.

Pronotum sehr unbedeutend breiter als der Kopf. Vorderrand stumpfwinkelig: konkav; Seitenränder beinahe parallel; Hinterrand schwach abgerundet, in der Mitte undeutlich eingebuchtet.

Länge des Körpers $6 \mathrm{~mm}$.

„ Kopfes mit Mandibeln $3 \mathrm{~mm}$.

, , , , ohne Mandibeln $1.8 \mathrm{~mm}$.

Breite des Kopfes $1 \cdot 24 \mathrm{~mm}$.

Nymphe. Körperlänge $6 \mathrm{~mm}$. Länge der Flügelscheiden $3 \mathrm{~mm}$.

Larve mit kleinsten Flügelscheiden. L. $5 \mathrm{~mm}$. Flügelscheiden kaum mehr als angedeutet.

"Arbeiter." Körperlänge 4,5 mm. Ohne Flügelscheiden.

Bemerkung. Wie ich schon in der Einleitung bemerkt habe, kommen die nächsten Verwandte dieser Art in Indien und zwar bei Singapore und Sarawak (Haviland) vor. Die indische Art (C. domesticus) ist aber viel mehr specializiert als $C$. fryeri und nähert sich sehr beträchtlich der amerikanischen Untergattung Cryptotermes (Banks).

Fundort. Aldabra: Takamaka, Fryer 6. X. 1908 (alle Stände).

\section{Untergattung GLyptotermes (Frogg.).}

Syn. Gattung Glyptotermes Froggatt.

Calotermes part, Desneux, Haviland.

3. Calotermes (Glyptotermes) scotti, n. sp.

Imago. Dunkelbraun mit hellerer Unterseite. Oberlippe gelblich. Beine graubraun. Flügel braun mit beinahe schwarzen Randrippen, narbig, irisierend. Antennen braun, mit helleren Basalgliedern. 
Mit äusserst schwacher Behaarung. Kopf und Thorax mit sehr dünnem Haarkleid. Abdomen besonders an den Hinterrändern der Segmentplatten mit Haaren oder Borsten.

Kopf dick, von oben gesehen rectangulär mit abgerundeten Ecken. Vorderrand des Transversalbandes gerade. Kopfnähte nicht sichtbar. Facettaugen ziemlich klein, flach. Punktaugen klein, nahe zu den Facettaugen gelegen. Clypeus kurz, beinahe gänzlich hyalin. Oberlippe gewölbt, stark geneigt. Antennen 12-gliedrig, nach aussen verdickt, von den Seiten ein wenig zusammengedrückt. 2. Glied ein wenig unbedeutend länger als 3. ; 3. ungefähr so lang wie 4. 12. Glied am längsten; 11. am dicksten. Palpen kurz. Oberkiefer verhältnismässig schwach.

Pronotum so breit wie der Kopf, vorn breit konkav; Seitenränder nach hinten schwach konvergierend, abgerundet. Hinterrand schwach abgerundet, in der Mitte höchst unbedeutend eingeschnitten. Hinterrand des Mesonotums gerade, des Metanotums schwach eingebuchtet.

Flügel relativ kurz; Membran dicht mit ziemlich grossen braunen Warzen oder Stacheln besetzt. Radius sector und mediana parallel ohne Seiten-ästen. Cubitus äusserst schwach hervortretend, mit zahlreichen Ästen sowohl zu dem Hinterrande des Flügels wie zu dem radius sector. Letztere besonders undeutlich. Die Äste sind nư durch die Lage der Stacheln angedeutet. Cerci kurz; Styli nur bei dem $\hat{\sigma}$ vorhanden.

Länge mit Flügeln $8-8 \cdot 2 \mathrm{~mm}$.

, ohne Flügel $5 \mathrm{~mm}$.

" des Kopfes vom Vorderrande des Transversalbandes $1 \mathrm{~mm}$.

Breite ,, , $0.9 \mathrm{~mm}$.

Soldat. Kopf gelbbraun, vorn ein wenig dunkler. Kiefer rotbraun, mit schwarzer Spitze. Körper strohgelb.

Behaarung wie bei der Imago. Abdominaltergite mit einer mittleren Borstenreihe, und einem hinteren Härchensaum. Sternite reichlicher aber unregelmässiger behaart.

Kopf rectangulär, beinahe cylindrisch, mit schwach abgerundeten Seiten und stark abgerundeten Hinterecken. Stirn ziemlich stark geneigt. Kopfnähte deutlich. Die Transversalnähte bilden medial einen deutlichen Winkel. Augen sehr klein, rudimentär. Mandibelkondylen deutlich, ein wenig von den Vorderecken der Antennleisten entfernt. Basalteil des Clypeus ziemlich schmal, sehr kurz. Spitzteil hyalin, wohlentwickelt. Oberlippe stark abgerundet, in der Mitte am breitesten. Antennen 11(?)-gliedrig. 1. Glied so lang wie die drei folgenden zusammengenommen; 2. so lang wie 4.; 3. kürzer. Mandibeln kurz, kräftig. Die linke mit zwei sehr schwachen, stumpfen Zähnen ein wenig vor der Mitte und mit einem kräftigen Basalzahn. Die rechte mit einem ziemlich kleinen, stumpfen Zahn an der Mitte und unmittelbar dahinten mit einem äusserst schwachen Zahn.

Pronotum von der Breite des Kopfes, so lang wie seine halbe Breite; vorn breit ausgerandet; Seitenränder abgerundet, nach hinten ein wenig konvergierend ; Hinterecken stark abgerundet; Hinterrand in der Mitte schwach eingebuchtet. Meso- und Metanotum in der Mitte eingeschnitten, Mesonotum mit schwachen Flügelansätzen. Hinterleib schmal. Beine kurz. 
Länge des Körpers $7 \mathrm{~mm}$.

, „, Kopfes mit Mandibeln $2 * 6 \mathrm{~mm}$.

Breite des Kopfes $1 \mathrm{~mm}$.

Nymphe: nicht vorhanden.

Junge Larve: Ohne Flügelanlagen. Körperlänge 2:5-3 mm.

Larve mit Flügelscheiden : Flügelscheiden seitwärts ausstehend. Körperlänge $3.5 \mathrm{~mm}$.

Larve mit Fliigelscheiden: Flügelscheiden schief nach hinten gerichtet. Körperlänge $4.5 \mathrm{~mm}$.

Larve mit Flïgelscheiden: Flügelscheiden kaum mehr als angedeutet. Körperlänge $5 \mathrm{~mm}$.

Larve mit Flïgelscheiden: Flügelscheiden ziemlich lang (mehr als $1 \mathrm{~mm}$.). Körperlänge $6.5 \mathrm{~mm}$.

“Arbeiter." Ohne Flügelscheiden. Körperlänge $6 \mathrm{~mm}$.

Bemerkungen. Die nächsten Verwandte dieser Art kommen in Indien, Australien und Süd-Amerika vor. Die Australischen Arten wurden von Froggatt zu einer eigenen Gattung Glyptotermes geführt. Diese Gattung zusammen mit Heterotermes* führte er zu einer eigenen Subfamilie Glyptotermitinae. Immerhin ist Glyptotermes morphologisch mit Calotermes so nahe verwandt, dass eine Trennung von dieser Gattung künstlich sein muss. Hingegen ist es auch kaum motiviert die Glyptotermes-Gruppe ganz einfach mit den übrigen Calotermes einzumischen, wie Desneux es gemacht hat.

Fundort. Seychellen. Silhouette: im Walde oberhalb Mare aux Cochons, mehr als 1000 Fuss oberhalb des Meeres, aus einem vermodernden gefallenen Palmenstamm, IX. 1908 (alle Stände).

4. Calotermes (Glyptotermes?) longus, n. sp.

Soldat. Kopf gelbbraun, vorn unbedeutend dunkler. Mandibeln schwarz, basal kaum heller. Körper strohgelb.

Kopf und Thorax dünn behaart. Hinterleib mit zwei transversalen jedoch nicht scharf ausgeprägten Reihen von Borsten und Haaren an jeder Platte.

Kopf lang, parallelseitig, mit den Mandibeln beinahe dreimal länger als breit, beinahe so hoch wie breit. Stirn ziemlich stark geneigt. Vorderecken rechteckig. Facettaugen sehr klein. Kopfnähte deutlich. Die Transversalnaht ist hinten bogenförmig konvex. Clypeus trapezförmig, mit grossem, vorn geraden Spitzteil. Oberlippe breit zungenförmig, stumpf. Antennenleisten ziemlich stark divergierend, mit einer schwach entwickelten, hinteren, ohrenförmigen Verbreitung. Antennen ?-gliedrig. 2. Glied unbedeutend länger als 3. und dicker als dieses; 3. Glied kurz, basal verschmälert; 4. ungefähr so lang wie 3. aber ein wenig breiter; 5. ein wenig länger. Mandibeln relativ kurz, aber kräftig. Die linke mit zwei deutlichen Zähnen vor der Mitte. Von diesen ist der hintere absatzförmig ausgezogen. Basalzahn gross, schneideförmig. Die rechte mit einem sehr kräftigen Zahn ungefähr in der Mitte oder unbedeutend hinter der Mitte. Basalzahn kräftig.

* Später wurde für Heterotermes die Subfanilie Heterotermitince aufgestellt. Ich glaube jedoch dass Heterotermes ganz einfach ein Leucotermes mit fehlenden Ozellen ist. (Bei L. tenuis fehlen Ozellen oft!) 
Pronotum einwenig breiter als der Kopf, transversal gewölbt, ein wenig länger als die halbe Breite, Vorderrand stumpfwinkelig konkav; Hinterrand unbedeutend eingebuchtet, Meso- und Metanotum schmäler als das Pronotum. Hinterleib lang, ziemlich schmal, gleich breit. Cerci klein. Styli wohl entwickelt. Beine ziemlich kurz.

Länge des Körpers $8 \mathrm{~mm}$.

, , Kopfes $3.7 \mathrm{~mm}$.

Breite des Kopfes $1.4 \mathrm{~mm}$.

Höhe des Kopfes $1 \cdot 2 \mathrm{~mm}$.

Bemerkungen. Ein relativ unbeschädigtes und ein sehr beschädigtes Exemplar vorhanden. Die Stellung dieser Art is sehr unsicher. Auf Grund der kurzen Oberkiefer habe ich sie zu Glyptotermes provisorisch geführt.

Fundort. Aldabra: Takamaka, 6. X. 1908, zusammen mit Procryptotermes fryeri von Fryer gefunden.

Fam. Mesotermitidæ, nov. fam.

Vorläufige Diagnose: Fontanell mit Fontanelldrüse bei Imagines und Soldaten vorhanden. Arbeiter mit Fontanell und Fontanellplatte. Praefrons dreieckig, Antennale in zwei Lateralhälften geteilt. Malpighische Gefässe 8. Hinterdarmabteilung I.-II., kurz.

Diese neue Familie umfasst folgende Subfamilien: Serritermitinæ, Termitogetoninæ, Coptotermitinæ, Leucotermitinæ und Rhinotermitinæ, alle mit Ausnahme der Rhinotermitinæ neu.

Subfam. Coptotermitinæ, n. subfam.

Gattung Arrhinotermes Wasm.

5. Arrhinotermes canalifrons (Sjöst.).

Syn. Termes? canalifrons Sjöst. Monograph. Nachtrag p. 47, 1904.

Procoptotermes canalifrons Holmgr. Termitenstudien, 1909.

Neotenisches Geschlechtsindividuum. Gelb, glänzend.

Beinahe vollständig unbehaart.

Kopf von oben beinahe cirkelrund. Fontanell gross, breit eiförmig. Kopfnähte als feine, weisse Linien sichtbar. Clypeus wohl entwickelt, ziemlich aufgetrieben; Hinterrand ziemlich stark konvex; Vorderrand schwach konvex. Basalteil längsgefurcht. Spitzteil schwach. Oberlippe stark geneigt, schalenförmig. Mandibeln wie bei Rhinotermes und Coptotermes. Antennen wahrscheinlich 22-gliedrig*. 2. Glied so lang wie 3. und 4. zusammengenommen. 3.-7. Glieder sämmtlich kürzer als 2., quer ; 8. beinahe kugelrund. Vom 5 an werden die Glieder allmählich länger.

Pronotum vorn schwach konkav, halbcirkelförmig, ein wenig schmäler als der Kopf. Mesonotum bedeutend breiter als Pronotum, mit sichelförmigen, ziemlich grossen Flügelansätzen. Metanotum ein wenig schmäler. Hinterleib wenig breiter als Mesonotum, von normaler Grösse.

* Auf einem Exemplar waren 21 Glieder vorhanden, ohne dass die Antenne jedoch vollständig war. 
Länge des Körpers $6 \mathrm{~mm}$.

" " Kopfes $1,5 \mathrm{~mm}$.

Breite des Kopfes $1,24 \mathrm{~mm}$.

, " Pronotums $1,1 \mathrm{~mm}$.

Länge des Pronotums $0,6 \mathrm{~mm}$.

Soldaten und Arbeiter stimmen gut mit den Beschreibungen Sjöstedt's überein. Sie besitzen jedoch beide Facettaugen, oft mit deutlichen Facetten.

Bemerkungen. Durch die Entdeckung der Neotenen dieser Art wurde ihre systematische Stellung bestimmt. Die Gattung Arrhinotermes wurde von Wasmann auf nur geflügelten Individuen gegründet. Die Soldaten wurden von Sjöstedt als Termes? canalifrons beschrieben, dessen Imagines unbekannt blieben. Für diesen Soldaten, welche unmöglich als Termes gelten könnten, schuf ich vorläufig die Gattung Procoptotermes. Durch Scott's Entdeckung dieser Neotenen wurde die Synonymie nachgewiesen, so dass Termes? canalifrons Sjöst. =Procoptotermes canalifrons (Sjöst.) Holmgr. = Arrhinotermes canalifrons (Sjöst.) Holngr. Die Gattung Arrhinotermes steht Coptotermes nahe, wie schon Desneux gegen Wasmann hervorgehoben hat, aber besitzt auch deutliche Verwandtschaftsbeziehungen zu Rhinotermes, wie es Wasmann ausgesprochen hat. Am besten kann man sich die Beziehungen dieser drei Gattungen vorstellen, wenn man annimmt, dass Arrhinotermes der gemeinsamen Stammform der beiden Sub-Familien Rhinotermitinæ und Coptotermitinæ nahe steht. Arrhinotermes gehört aber deutlich der Subfam. Coptotermitinæ, nimmt aber innerhalb dieser eine niedere Stufe ein.

Fundorte. Seychellen. Mahé: VII. 1908, Long Island (mit Neotenen). Praslin, XI. 1908 (Scott). Amiranten: Poivre, 10. X. 1905. Aldabra, 1908 (Fryer). Früher aus Tamatave, Madagascar bekannt (Mathiaux)*.

\section{Gattung CортотеRмes Wasm.}

\section{Coptotermes truncatus Wasm.}

Syn. Termes (Coptotermes) truncatus Wasmann, 1897; Desneux, 1904.

Eutermes truncatus (Wasm.) Sjöstedt, 1900, 1904.

Coptotermes truncatus Wasm., Holmgren, 1909.

Nymphen, Soldaten und Arbeiter liegen vor.

Bemerkungen. Mit dem Coptotermesindividuen liegt folgende Angabe vor: "From the bottom of a lighter (barge) in the harbour at Port Victoria (Mahé) : the workings of the Termites were mostly between the cross-beams and planks at the bottom of the lighter, and they had been submerged under several inches of salt water (which collects in the lighters) sometimes for more than a week at a time. Only one small part of the workings was above the level of the salt water." Dieser Bericht ist interessant aus dem. Gesichtspunkt der Verbreitungsbiologie der Art. Wenn so kräftige Kolonien, wie die fragliche (in der Kollektion giebt es Massen von Tieren von verschiedenen Kasten) in einer

* Nach Sjöstedt: Monographie, Nachtrag, 1907. 
Barke vorkommen kann, so ist natürlich eine Verbreitung durch Menschen von einer Lande zu einer anderen mit der Schiffsfahrt nicht ausgeschlossen Wenn diese Möglichkeit schon vom Beginn der Schiffsfahrt vorhanden war und eine Verbreitung mit Treibholz auch möglich sein muss, so muss es verwundern, dass thatsächlich so wenige Termitenarten zu den resp. Ländern eingeschleppt sind. Dies kann, glaube ich, nur dadurch erklärt werden, dass die Termiten in der Regel für plötzlich veränderte äussere Lebensbedingungen wenig widerstandsfähig sind. In diesem Verhältnis findet man vielleicht die Erklärung, dass Madagaskar und der naheliegende afrikanische Kontinent im Ganzen keine gemeinsame Termitenarten besitzen, obschon es wohl vorausgesetzt werden muss, dass Termitenaustausch während langen Zeiten zwischen den beiden Ländern vorkam. Und jedoch sind bisjetzt nur drei gemeinsame Arten bekannt: Termes bellicosus und natalensis und Calotermes madagascariensis. Bemerkt muss jedoch werden, dass die Termitenfauna von den nächst liegenden Teilen Afrikas nur sehr wenig bekannt ist.

Fundort. Seychellen. Mahé : X. 1908.

Fam. Metatermitidæ, nov. fam.

Vorläufige Diagnose: Fontanell mit Fontanellplatte bei Imagines und Arbeitern vorhanden. Soldaten mit Fontanell und Fontanelldrüse (mit wenigen Ausnahmen). Præfrons hinten gewöhnlich verbreitet. Antennale in zwei Lateralhälfte geteilt. Malpighische Gefässe 4-2. Hinterdarmabteilung I.-II. lang.

Diese Familie umfasst allen übrigen Termitengattungen, und kann in drei Serien oder Subfamilien vorläufig eingeteilt werden: Microcerotermitinæ, Termitinæ, und Eutermitinæ.

\section{[Subfam. Microcerotermitinæ, n. subfam.]}

Gattung Microcerotermes Silvestri.

7. Microcerotermes subtilis Wasm.

Syn. Termes subtilis Wasmann, 1897.

Eutermes subtilis (Wasm.) Sjöstedt, 1900, 1909, Desneux, 1904.

Microcerotermes subtilis Wasmann, 1903.

Imago: Die von Wasmann gegebene Beschreibung ist etwas unvollständig. Ich teile deshalb eine neue Beschreibung mit.

Kleine Art. Schwarzbraun, braun behaart. Clypeus gelbbraun. Mund und Körperunterseite sowie die zwei basalen Glieder der Antennen rostgelb. Tibien in der Mitte graubraun. Flügel schwarzbraun.

Kopf und Thorax sowie Abdominaltergite und Sternite ziemlich dicht behaart. Sternite ausserdem mit einer hinteren Borstenreihe.

Kopf breit oval, länger als breit. Facettaugen ziemlich klein, ein wenig ausstehend. Ozellen klein, von den Augen um einem Diameter entfernt. Fontanell sehr undeutlich. Clypeus hinten stark konvex. Basalteil gross, ziemlich aufgetrieben, vorn gerade, gerandet, an den Seiten abgerundet. Spitzteil deutlich, hyalin. Oberlippe zungenförmig. 
Antennen 14-gliedrig. 3. Glied äusserst kurz; 4. kürzer als 2. ; 5 . ein wenig länger als 4. beinahe so lang wie 2., breiter als dieses.

Pronotum deutlich schmäler als der Kopf ohne den Augen, so lang wie $\frac{2}{3}$ der Breite. Vorderrand gerade; Hinterrand ziemlich stark ausgeschnitten. Meso- und Metanotum hinten bogenförmig, schwach, ausgerandet.

Vordere Flügelschuppen ein wenig grösser als die hinteren. Flügel dreimal länger als breit, dicht mit kleinsten braunen Stacheln besetzt. Radius sector kräftig markiert. An den Vorderflügeln entspringt die mediana von der Schuppe, an den Hinterflügeln von dem radius sector. Sie ist an den Vorderflügeln einfach, an den Hinterflügeln gewöhnlich zweigeteilt. Cubitus mit 10-12 langen Zweigen.

Länge mit Flügeln $9 \mathrm{~mm}$.

„, ohne Flügel $5-6 \mathrm{~mm}$.

, des Kopfes $1 \mathrm{~mm}$.

Breite des Kopfes mit Augen 0,9 mm.

, , , , ohne Augen $0,8 \mathrm{~mm}$.

, , ,Prothorax $0,66 \mathrm{~mm}$.

Länge des Prothorax $0,4 \mathrm{~mm}$.

Königin. Länge des Körpers $17 \mathrm{~mm}$.

Breite des Körpers $5 \mathrm{~mm}$.

Soldaten und Arbeiter sind früher gut beschrieben. Vergl. Wasmann (1897) und Sjöstedt (1900, 1904).

Bemerkungen. Microcerotermes gehört zu den am weitesten verbreiteten Termitengattungen. Sie kommt in Afrika mit $3(-4)$ Arten, von denen eine $M$. palcarcticus (Sjöst.) sogar in Algier vorkommt, vor. Die übrigen M. parvus und fuscotibialis (Sjöst.) scheinen eine grosse Verbreitung zu haben. Auf Madagascar giebt es 2 Arten : M. subtilis und sicorce. In Indien treffen wir M. cylindriceps, und heimi Wasm., distans, dubius und serratus Havil. Australisch sind M. serratus und turneri Froggatt. Aus Neu-Guinea stammt M. birói Desn., aus Süd-Amerika M. struncki Sör und Bouvieri Desn. Nur die beiden Gattungen Calotermes und Eutermes können eine so weite Verbreitung aufweisen.

Fundort. Seychellen. Mahé : VII. 1908, Long Island, Königin und andere Stände in einem vermoderndem (gefallenen) Kokostamm VII. 1908.

[Subfam. Eutermitinæ, n. subfam.]

Gattung Eutermes (Hag) Fr. Müller.

8. Eutermes nigrita Wasm.

Nymphen, Soldaten und Arbeiter sowie Larven liegen vor.

Fundort. Seychellen. Silhouette, 1908, von einem Baumnest, ungefähr 2 Fuss oberhalb des Bodens (Scott). Praslin, XI. 1905, 5 getrocknete Soldaten. Früher aus Tanerive, Madagascar bekannt.

Mit wenig Reservation führe ich die folgende Imago zu dieser Art : Imago : Kopf und Hinterleib dunkel pechschwarz. Thorax viel heller braun. Clypeus und Basalglieder der 
Antennen sowie die Mandibeln heller. Oberlippe, Rest der Antennen und Beine graubraun. Flügel schwarzbraun.

Kopf ziemlich dicht behaart; Nacken ohne Haaren. Pronotum besonders an den Vorderecken und Seitenrändern ziemlich dicht behaart. Flügelschuppen und Randrippen dicht mit ziemlich langen Haaren besetzt. Behaarung des Hinterleibes ziemlich dicht.

Kopf ziemlich gross, flach gewölbt, breit oval mit grossen ziemlich ausstehenden Facettaugen. Ozellen mittelgross, mit ein wenig ausstehenden Rändern, um ihren halben Durchmesser von den Augen entfernt; zwischen den Ozellen läuft ein niedriger Wall. Kopfnähte nicht sichtbar. Fontanell sehr undeutlich, spaltförmig. Transversalband verhältnismässig lang. Basalteil des Clypeus kurz und breit, erreicht jedoch nicht den Seitenecken des Transversalbandes; Hinterrand ziemlich stark konvex; Vorderrand gerade; Seitenränder abgerundet; mit medialer Furche; Spitzteil wohl entwickelt. Oberlippe zungenförmig. Antennen 15-gliedrig. Basalglied dick. 2. Glied so lang wie 3.; 4. unbedeutend kürzer ; 5. ein wenig unbedeutend kürzer als 4. ; 6. so lang wie 4. Vom 5. nehmen die Glieder schwach in Länge zu.

Pronotum so breit wie der Kopf ohne Augen, so breit wie die doppelte Länge. Vorderrand gerade; Vorderecken stark niedergedrückt, abgerundet; Seitenränder stark nach hinten divergierend: Hinterrand ziemlich tief ausgeschnitten. Hinterrand des Mesonotums ein wenig tiefer und breiter ausgeschnitten als Metanotum.

Flügel ziemlich lang, dicht mit kleinsten Stacheln besetzt. Die mediana verläuft vie] näher dem cubitus als dem radius sector und besitzt in der Spitze ein paar kurze Zweige. Cubitus mit 10-12 schief gestellten Ästen, von denen die $7-8$ inneren kräftiger sind als die übrigen. Der dritte Zweig ist oft gabelig gespalten.

Länge mit Flügeln $15-16 \mathrm{~mm}$.

„, ohne Flügel $9 \mathrm{~mm}$. (getrocknet $7,5 \mathrm{~mm}$.).

, des Kopfes 1,9 mm.

Breite des Kopfes 1,73 mm.

Länge der Vorderflügeln $12-13 \mathrm{~mm}$.

Bemerkungen: In der Sammlung kommen 4 getrocknete Individuen vor, welche derselben Art angehören. Von diesen sind 2 von Gardiner auf Praslin, Seychellen 1905 gesammelt und 2 von der Seychellen Expedition auf Silhouette gefunden. Ferner kommen in der Sammlung 5 getrocknete Soldaten von Eutermes nigrita vor, welche von Gardiner 1905 auf Praslin gesammelt wurden, während auch von Eutermes nigrita Soldaten, Arbeiter und Nymphen aus Silhouette vorliegen. Dies macht es nun sehr wahrscheinlich, dass die Praslin-Imagines zu den Praslin-Soldaten und die SilhouetteImagines zu den Silhouette Individuen gehören. Diese Wahrscheinlichkeit wird nun zu beinahe vollständiger Gewissheit gesteigert, wenn wir den Körperbau der Imagines mit demjenigen der Nymphen vergleichen. Die Form des Kopfes, Lage der Ocellarflecke, Form des Prothorax ist dieselbe. Die Augen der Nymphen sind schon so gross, dass sie, wenn ausgewachsen, zu der Grösse derselben der Imagines gelangen müssen. Die unvollständige Färbung der Nymphen mit schon relatif dunklem Kopf, dunklen Flügelscheiden und beginnender Ausfärbung des Hinterleibes obschon das Thorax noch beinahe 
ungefärbt ist, deutet an, dass die Imagines, welche aus diesen Nymphen hervorgehen, die Färbung der fraglichen Imagines annehmen müssen.

Fundorte. Seychellen. Silhouette, 1908, Scott (2 Ex.). Praslin, XI. 1905 (2 Ex.).

9. Eutermes nigrita, subsp. mahéensis, n. subsp.

Soldat: unterscheidet sich von E. nigrita hauptsächlich in der Färbung, indem der Kopf rotbraun mit heller Nase und der Körper rostgelb-rostbraun ist. Der Kopf ist auch oft ein wenig grösser als bei nigrata und auch verhältnismässig breiter. Das Pronotum ist vorn in der Mitte nicht eingeschnitten. (Bei nigrita kommen aber nach Sjöstedt (1904) Exemplare vor, die ganzrandiges Pronotum besitzen.)

Arbeiter: Färbung im Ganzen wie bei den Soldaten. Kopf röthlich braun; Körper rostgelb-rostbraun. Der Kopf ist möglicherweise ein wenig breiter als bei E. nigrita. Die Antennen sind ähnlich gebaut, möglicherweise ein wenig kürzer.

Bemerkungen. Ich habe diese Individuen zufolge ihrer Färbung als selbständiger Subspecies aufgefasst. Über die Stellung von Eutermes nigrita nebst subsp. mahéensis unter den Eutermes-Arten will ich mich hier nicht näher äussern, obschon es mir scheint, als hätte die Art Anknüpfungspunkte zu der matangensis-Gruppe unter den indischen Termiten.

Fundort. Seychellen. Mahé, Long Island, VII. 1908.

\section{LITERATUR.}

Banks, N. Two new Termites. Entomol. News, Bd. xvii. 1906.

Desneux, J. Isoptera, Fam. Termitide in: Wütsman, Genera Insectorum, 1904.

Froggatt, W. W. Australian Termitidæ, Parts 1-3, Proc. Linn. Soc., N.S.W., 1895—97.

Haviland, G. D. Observations on Termites, etc., Journ. Linn. Soc., London, Zool., v. xxvi. 1898.

Holmgren, N. Madagassische Termiten gesammelt von Valter Kaudern. Arkiv for Zoologi, Bd. v. No. 13, 1909.

— Termitenstudien, Sv. Vet. Ak. Handl., Bd. xliv. No. 3, 1909.

SJöstedt, Y. Monographie der Termiten Afrikas, Sv. Vet. Ak. Handl., Bd. xxxiv. No. 4, 1900.

— Nachtrag, Ibid. Bd. xxxviii. No. 4, 1904.

Wasmann, E. Einige neue Termiten aus Ceylon und Madagascar, etc., Wien. entomol. Ztg., 1893.

_ Termiten von Madagascar und Ost-Afrika. Abh. Senckenb. naturf. Ges. Frankfurt, v. xxi. 1897.

Termiten, Termitophilen und Myrmekophilen, gesammelt auf Ceylon, etc., Zool. Jahrb. Abth. für Syst., Bd. xvii. 1903. 


\section{$2 \mathrm{BHL}$ Biodiversity Heritage Library}

Holmgren, Nils F . 1910. "The Percy Sladen Trust expedition to the Indian ocean in 1905. No. VIII.-Isoptera." Transactions of the Linnean Society of London 14, 135-148. https://doi.org/10.1111/j.1096-3642.1910.tb00527.x.

View This Item Online: https://www.biodiversitylibrary.org/item/55334

DOI: https://doi.org/10.1111/j.1096-3642.1910.tb00527.x

Permalink: https://www.biodiversitylibrary.org/partpdf/193237

\section{Holding Institution}

MBLWHOI Library

\section{Sponsored by}

Biodiversity Heritage Library

\section{Copyright \& Reuse}

Copyright Status: NOT IN COPYRIGHT

This document was created from content at the Biodiversity Heritage Library, the world's largest open access digital library for biodiversity literature and archives. Visit BHL at https://www.biodiversitylibrary.org. 